\title{
Resiliência em Médicos: Contributos para a Validação da Versão Portuguesa da Escala de Resiliência
}

\section{Resilience in Physicians: Contributions to the Validation of the European Portuguese Version of the Resilience Scale}

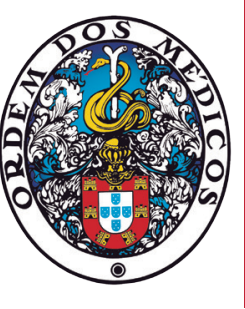

\author{
Carla SERRÃO $\square^{1,2}$, Luísa CASTRO ${ }^{3,4,5}$, Andreia TEIXEIRA ${ }^{3,4,6}$, Ana Rita RODRIGUES ${ }^{7,8}$, Ivone DUARTE ${ }^{3,4}$ \\ Acta Med Port 2021 Jul-Aug;34(7-8):523-532 - https://doi.org/10.20344/amp.14657
}

\section{RESUMO}

Introdução: Este estudo visa explorar a validação da Escala de Resiliência (25 itens e 14 itens), nas suas versões longa e breve. Este instrumento avalia a capacidade de o indivíduo suportar os fatores de stress, de prosperar e dar sentido a desafios vitais.

Material e Métodos: A amostra integrou 511 médicos portugueses. Ambas as versões foram validadas através do estudo de validade de estrutura interna, de fiabilidade e de validade convergente. A validade de estrutura interna foi analisada através da técnica da análise de componentes principais. A fiabilidade foi verificada pelo estudo de consistência interna. Para a validade convergente, calculou-se os coeficientes de correlação entre estas versões da Escala de Resiliência e outras escalas validadas para medir depressão, ansiedade, stress e satisfação com a vida.

Resultados: Ambas as versões da Escala de Resiliência apresentaram boa consistência interna. Para cada uma das versões, optou-se pela análise de componentes principais a um fator. A validade convergente foi verificada por correlações positivas significativas entre a Escala de Resiliência 25 e Escala de Resiliência 14 e uma escala de satisfação com a vida e por correlações negativas significativas entre as duas versões da Escala de Resiliência e as subescalas de depressão, ansiedade e stress.

Discussão: Os resultados evidenciaram o caráter unidimensional das duas versões da Escala de Resiliência e apoiam a sua utilidade e validade na classe dos médicos.

Conclusão: Trata-se do primeiro estudo de validação desta escala num grupo de médicos. Os seus resultados são muito satisfatórios, recomendando-se o uso deste instrumento neste grupo específico.

Palavras-chave: Inquéritos e Questionários; Médicos; Portugal; Psicometria; Resiliência Psicológica

\section{ABSTRACT}

Introduction: The aim of this study is to explore the validation of the Resilience Scale in its long and brief versions (25 items and 14 items). This instrument assesses the individual's ability to withstand stressors, thrive and make sense of vital challenges.

Material and Methods: The sample included 511 Portuguese physicians. Both versions were validated through the study of internal structure validity, reliability, and convergent validity. The validity of the internal structure was analysed using the principal component analysis technique. Reliability was verified by the internal consistency study. For convergent validity, the correlation coefficients between these versions of the Resilience Scale and other scales validated to measure depression, anxiety, stress, and life satisfaction were calculated.

Results: Both versions of Resilience Scale showed good internal consistency. For each of the versions, one factor was retained in the principal component analysis. Convergent validity was verified by significant positive correlations between Resilience Scale ( 25 and 14) and a life satisfaction scale and significant negative correlations between Resilience Scale and depression, anxiety, and stress subscales.

Discussion: The results show the one-dimensional character of both versions of the Resilience Scale and support their usefulness and validity in the physician's class.

Conclusion: This is the first validation study of this scale in a group of physicians. Its results are very satisfactory, and its use in this specific group is recommended.

Keywords: Physicians; Portugal; Psychometrics; Resilience, Psychological; Surveys and Questionnaires

\section{INTRODUÇÃo}

A emergência do paradigma salutogénico tem dado origem a estudos sobre fatores e mecanismos de proteção da saúde mental. ${ }^{1}$ Nesta tendência, a resiliência tem sido um dos construtos mais investigados. ${ }^{2,3}$

A resiliência é definida pela Associação de Psicólogos Americana como o processo de adaptação positiva a adversidades, traumas, tragédias, ameaças ou fontes signifi1. Escola Superior de Educação. Politécnico do Porto. Porto. Portugal.

2. INED - Centro de Investigação em Educação. Escola Superior de Educação. Politécnico do Porto. Porto. Portugal.

3. MEDCIDS - Departamento de Medicina da Comunidade, Informação e Decisão em Saúde. Faculdade de Medicina. Universidade do Porto. Porto. Portugal.

4. CINTESIS - Centro de Investigação em Tecnologias e Serviços de Saúde. Faculdade de Medicina. Universidade do Porto. Porto. Portugal.

5. Escola Superior de Saúde. Politécnico do Porto. Porto. Portugal.

6. Instituto Politécnico de Viana do Castelo. Viana do Castelo. Portugal.

7. Departamento de Ciências Sociais e do Comportamento. Instituto Universitário da Maia. Maia. Portugal.

8. Centro de Psicologia da Universidade do Porto. Porto. Portugal.

$\triangle$ Autor correspondente: Carla Serrão: carlaserrao@ese.ipp.pt

Recebido: 30 de julho de 2020 - Aceite: 24 de setembro de 2020 - First published: 22 de fevereiro de 2021 - Online issue published: 01 de julho de 2021 Copyright $\odot$ Ordem dos Médicos 2021 cativas de stress. ${ }^{4}$ Embora essa definição seja útil, alguns autores $^{5}$ sustentam que a mesma não reflete a complexa natureza da resiliência. Para os autores, os determinantes do construto incluem uma série de fatores biológicos, psicossociais e culturais que interagem entre si para determinar como é que a pessoa responde a experiências de stress. A resiliência diz respeito à capacidade humana para 
enfrentar situações de risco e recuperar o equilíbrio socio-emocional ${ }^{6}$ envolvendo crenças, comportamentos, pensamentos e ações, ${ }^{4}$ sendo considerada uma variável que promove a adaptação individual e modera os efeitos negativos do stress. ${ }^{7}$ Nesta perspetiva, a resiliência é um processo psicológico que pode ser desencadeado em determinados momentos de vida, sendo entendida como uma interação dinâmica entre as características individuais e a complexidade dos contextos. ${ }^{5,8}$

A resiliência tem sido analisada não só em momentos normativos desenvolvimentais de transição (e.g., entrada na escola, processo de preparação para a parentalidade), como também em situações de crise não normativas, como o desemprego ${ }^{9}$ e situações severas de stress, na sequência de acidentes naturais ou terrorismo, por exemplo. ${ }^{5}$ Nesta ordem de ideias, a resiliência é caracterizada como sendo um construto mutável e dinâmico, em que os sujeitos não são considerados como resilientes, mas sim como estando resilientes, ${ }^{10,11}$ sendo capazes de utilizar recursos intrapessoais e interpessoais para enfrentar de forma positiva a situação de stress/risco. ${ }^{5,12}$

O estudo da resiliência parece mais pertinente quando aplicado a ambientes que pressupõem a existência de vulnerabilidade e que exigem e apresentem características adaptativas, ${ }^{2}$ como é o caso das instituições de saúde e dos profissionais de saúde, dadas as contínuas e significativas situações de stress. Atualmente, tem vindo a verificar-se um crescente interesse específico na classe profissional de médicos. ${ }^{13-15}$ Esta tendência pode justificar-se pelo facto de a medicina se constituir como uma carreira gratificante e, ao mesmo tempo incrivelmente exigente, originando níveis elevados de ansiedade, ${ }^{16,17}$ stress, depressão, burnout ${ }^{15,16,18}$ e outros problemas físicos, nomeadamente, processos inflamatórios crónicos que, por sua vez, podem originar arteriosclerose e doenças cardiovasculares. ${ }^{19-21}$

De facto, os profissionais de saúde, e em particular os médicos, lidam quotidianamente com fontes múltiplas de stress associadas à natureza da sua profissão. Entre eles destacam-se a excessiva carga de trabalho e os horários de trabalho exigentes (turnos com trabalho noturno), originando, não raras vezes, privação de sono. ${ }^{22}$ As múltiplas funções que desempenham, ${ }^{23,24}$ as questões organizacionais associadas à comunicação e interação com outros profissionais, os dilemas clínicos, os conflitos com doentes, ${ }^{25}$ a complexidade da relação médico-doente, ${ }^{22}$ o contacto persistente com o sofrimento e a dor, constituem um leque variado de stressores. Esta exposição recorrente pode afetar o bem-estar psico-emocional e físico e ter como consequência a exaustão, ${ }^{26}$ mas também pôr em causa o processo profissional cuidativo. ${ }^{27}$

A exposição a fatores de risco não prevê de forma definitiva uma adaptação negativa. Contudo, quando o número de fatores de risco (individuais e ambientais) é maior que os fatores de proteção, os indivíduos podem, perante o cumulativo de novas situações de pressão, desenvolver exaustão e sintomatologia física e emocional. ${ }^{28}$

Diversos estudos desenvolvidos com profissionais de saúde descrevem a resiliência como um fator de proteção ao burnout, ${ }^{29}$ e uma variável amortecedora do impacto dos efeitos negativos dos stressores ocupacionais..$^{30,31}$

Embora não exista uma escala de resiliência gold standard, ${ }^{32}$ a Escala de Resiliência - ER (Resilience Scale) de Wagnild e Young ${ }^{7,33}$ foi a primeira escala a ser desenvolvida, e é uma das mais utilizadas na investigação. ${ }^{34}$

No final dos anos 80, Wagnild e Young realizaram um estudo qualitativo no qual entrevistaram 24 mulheres idosas que se adaptaram com sucesso a situações de vulnerabilidade. A análise das entrevistas possibilitou a construção da ER. Este instrumento é de autorrelato, composto por 25 itens. Os scores variam de 25 a 175 pontos, com os mais elevados a indicarem alto grau de resiliência. O score médio para a ER foi de 147,91 pontos (DP $=16,85$ pontos). ${ }^{7}$ Um score abaixo de 121 pontos é considerado como indicativo de 'reduzida resiliência'; um resultado entre 121 e 145 pontos é considerado como 'resiliência moderada'; e acima dos 145 pontos é considerado de 'resiliência elevada'. ${ }^{7}$ A fiabilidade da escala evidenciou-se elevada, com um alfa de Cronbach $(\alpha)$ de 0,91 , e as correlações item-total corrigidas variaram de 0,37 a 0,75 , com a maioria a pontuar entre 0,50 e 0,70. A análise em componentes principais (ACP) com rotação varimax evidenciou cinco componentes: serenidade, perseverança, autoconfiança, sentido de vida e autossuficiência. ${ }^{33}$

Após este primeiro trabalho, Wagnilg e Young ${ }^{33}$ conduziram vários estudos com diferentes amostras (e.g., estudantes de ensino superior, estudantes de pós-graduação, cuidadores de pessoas com demência de Alzheimer, mães nulíparas a regressar ao trabalho), ${ }^{35}$ tendo-se confirmado a fiabilidade e validade do instrumento. No entanto, encontraram ambiguidades na interpretação para 3, 4 e 5 fatores. O gráfico de escarpa mostrou o ponto de corte entre os fatores 1 e 2 e os restantes, e a solução fatorial indicou dois fatores substanciais. Estes resultados levaram as autoras a optar pela solução a dois fatores, com pesos acima de 0,40 para cada item, considerando que refletem a definição teórica de resiliência e suportam a validade de construto da ER. O fator 1 ('competência pessoal') é constituído por 17 itens e integra qualidades como autoconfiança, perseverança, independência, determinação e invencibilidade; e o fator 2 ('aceitação do eu e da vida') é constituído por oito itens e integra características como equilíbrio, flexibilidade e perspetiva equilibrada da vida. Estes fatores explicaram $44 \%$ da variância total. ${ }^{7}$ Este instrumento avalia a capacidade de o indivíduo suportar os fatores de stress, de prosperar e dar sentido a desafios vitais.

Wagnild $^{36}$ realizou uma revisão da literatura sobre a ER e identificou a sua tradução e validação em mais de doze países. A sua validade foi analisada junto de vários grupos populacionais e etários. Nestes estudos, os valores de a variaram entre 0,72 e 0,94, atestando boa consistência interna. $^{36}$

A ER, além da versão longa de 25 itens - ER25, apresenta uma versão breve com 14 itens - ER14 ${ }^{35}$ (itens 2, 6, 7 , $8,9,10,13,14,15,16,17,18,21$ e 23). AACP com rotação 
direta oblimin revelou a existência de um fator, responsável por explicar $53 \%$ da variância total, indicando uma dimensão subjacente comum. O a foi de 0,93. A ER14 correlacionou-se fortemente com a ER25 ( $r=0,97, p<0,001)$ e moderadamente com uma medida de avaliação de sintomas depressivos $(r=-0,41)$ e uma medida de satisfação com a vida $(r=0,37) \cdot{ }^{35}$

Tomando como referência estudos recentes da ER (versões longa e breve), e embora Wagnild e Young ${ }^{7}$ tivessem originalmente abordado a multidimensionalidade desta escala, a ER é vulgarmente utilizada com uma pontuação global. ${ }^{35,37,38}$

Em contexto português foram encontradas três validações da ER. Uma da autoria de Felgueiras et al, ${ }^{39}$ partindo de uma amostra de 215 adolescentes entre os 10 e 16 anos; uma de Oliveira e Machado, ${ }^{40}$ com uma amostra de 451 estudantes de ensino superior entre os 18 e os 26 anos; e outra de Deep e Leal, ${ }^{41}$ com uma amostra de 365 indivíduos entre os 18 e os 83 anos.

Felgueiras et $a^{\beta 9}$ concluíram que a ER tinha indicadores satisfatórios de fiabilidade e estabilidade para 24 itens (foi excluído o item 5), obtendo um a de 0,82 e uma correlação de teste-reteste de $0,73(p<0,001)$. A validade de estrutura interna foi estudada pela ACP com rotação varimax, tendo identificado cinco fatores não homogéneos. Embora tenha explicado $46 \%$ da variação total, nenhum dos fatores correspondiam à proposta teórica de Wagnild e Young. ${ }^{7}$ Verificaram também que os itens 6 e 11 apresentaram baixos pesos fatoriais $(<0,40)$, o item 13 apresentou um peso negativo no seu fator e o fator 'autossuficiência' tinha apenas dois itens. Os itens nomeados apresentaram correlações baixas com a escala total e, se removidos, aumentariam a fiabilidade total da ER.

Também Oliveira e Machado, ${ }^{40}$ no estudo das propriedades métricas da $\mathrm{ER}$, concluíram que se tratava de um instrumento fiável $(\alpha=0,89)$, válido e sensível. A análise fatorial forçada a dois fatores, de acordo com a proposta das autoras do instrumento, ${ }^{7}$ explicou apenas $37,8 \%$ da variância total. Neste sentido, Oliveira e Machado ${ }^{40}$ procederam a uma ACP com rotação varimax, tendo obtido uma distribuição dos itens por cinco fatores que explicavam 52,5\% da variância total.

Por fim, Deep e Leal, ${ }^{41}$ no estudo das propriedades métricas da ER, verificaram que as análises iniciais sugeriam a existência de seis fatores que explicavam $56,7 \%$ da variância. No entanto, seguiram o procedimento da escala original e obtiveram quatro fatores, tendo sido eliminados dois itens (itens 1 e 7 ), com uma variância total explicada de $47,2 \%$, e com um $\alpha$ de 0,87 para 23 itens.

$\mathrm{O}$ crescente interesse no estudo da resiliência enquanto variável protetora e de recuperação da saúde tem refutado a necessidade de desenvolver escalas de avaliação que garantam a sua validade. ${ }^{42}$ Por isso, a avaliação da qualidade destes instrumentos junto de populações específicas, como é o caso dos médicos, é de importância fundamental. Além disso, a medicina é das disciplinas de saúde menos investigadas a este nível.
Embora o estudo da ER seja amplo, e permita confirmar a boa consistência interna do instrumento, permanecem algumas controvérsias em relação à sua estrutura fatorial.

Perante os argumentos levantados, o objetivo deste estudo é contribuir para a validação da ER, nas versões longa e breve, quando aplicadas a médicos portugueses.

\section{MATERIAL E MÉTODOS}

Este é um estudo transversal, quantitativo, de natureza analítica, baseado na aplicação de um questionário online, partilhado por bola de neve pelas redes sociais com o apoio de instituições de saúde e organizações profissionais. Este questionário foi composto por uma secção sociodemográfica e profissional e por uma bateria de escalas. A amostra foi recolhida por conveniência, entre 9 de maio e 8 de junho de 2020, e integrou 511 médicos residentes em Portugal e Ilhas.

O estudo teve início após parecer positivo da Comissão de Ética da Faculdade de Medicina da Universidade do Porto (referência n. ${ }^{\circ}$ 184/2020, de 7 de maio de 2020).

O objetivo principal deste estudo é a validação da ER25 (versão longa, ${ }^{7}$ versão portuguesa) ${ }^{40}$ e da ER14 (versão breve). ${ }^{35}$ Esta validação consistiu em três vertentes: validação da estrutura interna, estudo de fiabilidade e análise de validade convergente. Dada a falta de consenso na literatura sobre o número de fatores da $\mathrm{ER}$, a validação da estrutura interna foi realizada através da ACP com rotação varimax. Para o número de fatores a extrair foram considerados três critérios: fatores com valor próprio superior a 1 (método de Kaiser), observação do gráfico de escarpa, e fatores com percentagem de variância explicada superior a $5 \%$. Para averiguar se a amostra é adequada à ACP, calculou-se a medida de Keiser-Meyer-Olkin (KMO) e o teste de esfericidade de Bartlett. ${ }^{43}$

A fiabilidade das escalas foi verificada através da consistência interna, pelo cálculo do coeficiente alfa de Cronbach $(\alpha),{ }^{44}$ da média das correlações entre itens, e da correlação item-total corrigida. Valores de a acima de 0,7 são considerados aceitáveis. ${ }^{42,45} \mathrm{~A}$ média das correlações entre itens deve variar entre 0,15 e 0,5, para garantir que medem o mesmo construto e, por outro lado, não serem redundantes. ${ }^{46}$ Cada item deve correlacionar-se com o total do construto (correlação item-total corrigida) ${ }^{42}$ com valores entre 0,3 e 0,7. Efeitos de 'teto' ou 'chão' estão presentes quando mais do que $15 \%$ dos respondentes atingem o máximo ou o mínimo teórico da escala, respetivamente. A existência destes efeitos limita a validade da escala. ${ }^{47}$

Relativamente à validade convergente da ER, foram calculadas correlações com escalas que teoricamente estão relacionadas com a resiliência, tal como identificado pelas autoras da ER original: DASS-depressão, DASS-ansiedade, DASS-stress e Satisfação com a Vida. ${ }^{35}$

A Depression Anxiety Stress Scale (DASS) ${ }^{48,49}$ é constituída por 21 itens e organiza-se em três subescalas: depressão, ansiedade e stress. Cada subescala é constituída por sete itens. Os sujeitos avaliam a extensão em que experimentaram cada sintoma durante a última semana, 
numa escala de quatro pontos (0: "não se aplicou nada a mim", 1: "aplicou-se a mim algumas vezes", 2: "aplicou-se a mim muitas vezes" e 3: "aplicou-se a mim a maior a parte das vezes"). Os resultados de cada subescala são determinados pela soma das pontuações dos sete itens, variando entre 0 e 21 pontos. As pontuações mais elevadas de cada subescala correspondem a estados afetivos mais negativos. A DAAS na versão portuguesa ${ }^{49}$ apresentou boa consistência interna (depressão: $\alpha=0,85$; ansiedade: $\alpha=$ 0,74 e stresse: $\alpha=0,81$ ).

A escala de Satisfação com a Vida ${ }^{50,51}$ visa avaliar a componente cognitiva do bem-estar subjetivo, e é constituída por cinco itens. Cada item é uma afirmação à qual o inquirido tem de atribuir um nível de concordância, através de uma escala de sete pontos (de 1: "discordo muito" a 7 : "concordo muito"). A escala foi adaptada para a população portuguesa, pela primeira vez por Neto et $a^{52}(\alpha=0,78)$. Simões ${ }^{51}$ repetiu a validação da escala, reduzindo a amplitude de resposta de sete para cinco pontos (de 1: "discordo muito" a 5: "concordo muito"), obtendo um valor de a de 0,77 . O resultado da escala é determinado pela soma das pontuações dos cinco itens, variando de cinco a 25 pontos. Pontuações elevadas sugerem uma maior satisfação com a vida. ${ }^{51}$ Esta escala é caraterizada por consistência interna aceitável e alta (versão original: $\alpha=0,87$ e versão em português: $\alpha=0,77) \cdot{ }^{50,51}$

A análise de dados foi realizada utilizando o pacote de software estatístico SPSS, versão 26.0 para Windows (IBM SPSS Inc.). As variáveis categóricas foram descritas por frequências absolutas e relativas, $n(\%)$. A normalidade das variáveis foi verificada por observação dos histogramas. As correlações foram calculadas através do coeficiente de correlação de Spearman, $r$.

Valores de $p \leq 0,05$ foram considerados significativos.

\section{RESULTADOS}

Participaram no estudo 511 médicos (Tabela 1). O grupo era constituído maioritariamente por mulheres $(79,8 \%)$ e a mediana $\left(Q_{1}-Q_{3}\right)$ das idades foi de 35 (29-43) anos. A maioria dos médicos estava casado ou em união de facto $(42,9 \%)$ e sem filhos $(57,7 \%)$. Relativamente ao nível de escolaridade, $62,6 \%$ dos participantes tinham mestrado. Cerca de $30 \%$ tinha entre um a cinco anos de experiência profissional, seguidos de $25,4 \%$ com mais de 16 anos e $24,1 \%$ entre seis a 10 anos. Os cuidados de saúde primários foi o sector mais frequente, representando cerca de $44 \%$ dos profissionais da amostra. É de salientar ainda que alguns participantes desenvolviam a sua atividade em diferentes setores. A descrição das escalas aplicadas pode também ser consultada na Tabela 1.

\section{Validação da ER25 \\ Consistência interna}

$\mathrm{Na}$ ER25, o coeficiente $\alpha$ obtido foi elevado ( $\alpha=0,937)$, aumentando com a exclusão dos itens 11 e 12 (Tabela 2). A média de correlações entre itens foi de 0,391 , pelo que está entre 0,15 e 0,5 . As correlações item-total corrigidas variam entre 0,320 (item 11) e 0,737 (item 17), mas a maioria está situada entre 0,5 e 0,7 .

Em relação aos efeitos de 'teto' ou 'chão', 0\% dos participantes pontuaram o mínimo da escala e apenas 0,4\% tiveram a pontuação máxima, o que evidencia a validade da versão portuguesa da escala para a população de médicos.

\section{Validade da estrutura interna}

Os dados obtidos revelaram-se adequados para uma $\mathrm{ACP}(\mathrm{KMO}=0,943 ; p<0,001)$. Na determinação do número de fatores a reter, o método de Kaiser indicou quatro fatores, que juntos explicam cerca de $59,3 \%$ da variância total dos dados; o gráfico de escarpa sugere a extração de três fatores, que explicam $55,2 \%$ da variância; e o critério da percentagem de variância sugere três fatores. Uma vez que dois dos três critérios aplicados indicaram a extração de três fatores, essa será a estrutura analisada na Tabela 3.

Nesta estrutura de três fatores, a comunalidade mais baixa foi de $41,7 \%$ (item 14). Como é possível observar na Tabela 3, existem vários itens a saturar simultaneamente em dois fatores com pesos superiores a 0,4 , como é o caso dos itens 4, 5, 8, 9, 15, 17, 21, 23 e 25, criando ambiguidade na interpretação. Além disso, a opção de associar o item ao fator no qual apresenta um peso mais elevado não vai ao encontro do que é mencionado na literatura nem constitui uma distribuição lógica de fatores, pelo que se optou por forçar a fatorização a um fator (pelo critério do gráfico de escarpa).

A ACP com um fator explicou cerca de $42,6 \%$ da variância total e é o item 11 que apresenta a comunalidade mais baixa $(10,7 \%)$. Os pesos fatoriais variaram entre 0,326 e 0,776 (para os itens 11 e 10, respetivamente), como se pode verificar na coluna A da Tabela 4. Tendo em conta que, na análise da consistência interna, a exclusão do item 11 aumentaria o valor de a e, nesta estrutura, é o item que apresenta a comunalidade mais baixa, optou-se por excluir o item 11 e voltar a aplicar a ACP a um fator. Esta nova estrutura unidimensional explicou um total de $43,98 \%$ da variância total. Neste caso, é o item 12 que apresenta a comunalidade mais baixa $(15,8 \%)$. A saturação fatorial dos itens variou de 0,397 (item 12) a 0,776 (item 10), como é possível observar na coluna $\mathrm{B}$ da Tabela 4. O valor de a para estes 24 itens foi de 0,939. Mais uma vez, tendo em conta que, na análise da consistência interna, a exclusão do item 12 aumentaria o valor de a e, nesta estrutura, este item apresenta uma comunalidade muito baixa, optou-se por excluir também o item 12 e voltar a aplicar a ACP a um fator. Esta nova estrutura unidimensional explicou um total de $45,27 \%$ da variância total. Neste caso, é o item 22 que apresenta a comunalidade mais baixa (23,7\%). A saturação fatorial dos itens variou de 0,486 (item 22) a 0,780 (item 10), como é possível observar na coluna $\mathrm{C}$ da Tabela 4 . O valor de a para estes 23 itens foi de 0,941 . 
Tabela 1 - Características sociodemográficas dos indivíduos e medidas sumárias das Escalas de Resiliência (ER25 e ER14), Depressão, Ansiedade e Stress (DASS) e Satisfação com a Vida, aplicadas na amostra de 511 médicos.

\begin{tabular}{|c|c|c|}
\hline \multicolumn{3}{|l|}{ Variáveis } \\
\hline \multirow[t]{3}{*}{ Sexo, n(\%) } & Mulheres & $408(79,8)$ \\
\hline & Homens & $102(20,0)$ \\
\hline & Outro & $1(0,2)$ \\
\hline \multirow[t]{5}{*}{ Idade em anos, $\mathrm{n}(\%)$} & Menos de 35 anos & $255(49,9)$ \\
\hline & Entre 35 e 44 anos & $141(27,6)$ \\
\hline & Entre 45 e 54 anos & $57(11,2)$ \\
\hline & Entre 55 e 64 anos & $50(9,8)$ \\
\hline & Acima de 65 anos & $8(1,6)$ \\
\hline \multirow[t]{4}{*}{ Estatuto conjugal, $n(\%)$} & Solteiro & $219(42,9)$ \\
\hline & Casado/união de facto & $257(50,3)$ \\
\hline & Divorciado/separado & $33(6,5)$ \\
\hline & Viúvo & $2(0,4)$ \\
\hline \multirow[t]{2}{*}{ Tem filhos, $n(\%)$} & $\operatorname{Sim}$ & $216(42,3)$ \\
\hline & Não & $295(57,7)$ \\
\hline \multirow[t]{4}{*}{ Nível educacional, n(\%) } & Licenciatura & $164(32,1)$ \\
\hline & Pós-Graduação/Especialista & $5(1,0)$ \\
\hline & Mestrado & $320(62,6)$ \\
\hline & Doutoramento & $22(4,3)$ \\
\hline \multirow[t]{5}{*}{ Anos de experiência profissional, $n(\%)$} & Menos de 1 ano & $25(4,9)$ \\
\hline & De 1 a 5 anos & $153(29,9)$ \\
\hline & De 6 a 10 anos & $123(24,1)$ \\
\hline & De 11 anos a 15 anos & $80(15,7)$ \\
\hline & Mais de 15 anos & $130(25,4)$ \\
\hline \multirow[t]{11}{*}{ Setores de trabalho, $\mathrm{n}(\%)$} & Unidade Cuidados Intensivos & $39(7,6)$ \\
\hline & Serviço de urgência & $176(34,4)$ \\
\hline & Emergência intra-hospitalar & $21(4,1)$ \\
\hline & Emergência extra-hospitalar & $12(2,3)$ \\
\hline & Cuidados de Saúde Primários & $225(44,0)$ \\
\hline & Serviço internamento & $189(37,0)$ \\
\hline & Unidade Cuidados Primários & $17(3,3)$ \\
\hline & Bloco operatório & $66(12,9)$ \\
\hline & Sector privado & $66(12,9)$ \\
\hline & Desempregado & $2(0,4)$ \\
\hline & Reformado & $2(0,4)$ \\
\hline \multicolumn{2}{|l|}{ ER25, M $\pm S D ;$ Med $\left[Q_{1}, Q_{3}\right] ;$ min-máx } & $131,2 \pm 20,6 ; 134[122,145] ; 37-175$ \\
\hline \multicolumn{2}{|c|}{ ER14, $M \pm S D ; M e d\left[Q_{1}, Q_{3}\right] ;$ min-máx } & $76,7 \pm 12,4 ; 79[70,85] ; 22$ - 98 \\
\hline \multicolumn{2}{|c|}{ DASS-depressão, $M \pm S D$; Med $\left[Q_{1}, Q_{3}\right]$; min-máx } & $3,9 \pm 4,4 ; 2[1,6] ; 0-21$ \\
\hline \multicolumn{2}{|c|}{ DASS-ansiedade, $\mathrm{M} \pm \mathrm{SD}$; Med $\left[\mathrm{Q}_{1}, \mathrm{Q}_{3}\right]$; min-máx } & $2,8 \pm 3,4 ; 2[0,4] ; 0-17$ \\
\hline \multicolumn{2}{|l|}{ DASS-stress, $M \pm S D$; Med $\left[Q_{1}, Q_{3}\right]$; min-máx } & $7,2 \pm 4,6 ; 6[4,10] ; 0-21$ \\
\hline \multicolumn{2}{|c|}{ Satisfação com a Vida, $M \pm S D$; Med $\left[Q_{1}, Q_{3}\right]$; min-máx } & $17,1 \pm 4,1 ; 18[14,20] ; 5-25$ \\
\hline
\end{tabular}

\section{Validação da ER14}

Foram conduzidas as mesmas análises psicométricas para a ER14.

\section{Consistência interna}

$\mathrm{Na}$ ER14, o coeficiente $\alpha$ também foi elevado ( $\alpha=$ $0,923)$. A média das correlações entre itens foi 0,467 , pelo que está entre 0,15 e 0,5. As correlações item-total corrigidas variam entre 0,536 (item 7) e 0,751 (item 10), com a 
maioria situada entre 0,6 e 0,7 .

\section{Validação da estrutura interna}

Os dados obtidos revelaram-se adequados para uma $\mathrm{ACP}(\mathrm{KMO}=0,941 ; p<0,001)$. Quanto à extração de fatores pela ACP, o gráfico de escarpa concordou com o método de Kaiser, sugerindo a retenção de apenas um fator, responsável por explicar cerca de $50,9 \%$ da variância total. O critério da percentagem de variância sugeriu reter quatro fatores, que explicavam cerca de 69,2\% da variância dos dados. Dado que dois dos três critérios indicam a extração de um fator, essa será a estrutura proposta, indicando um construto subjacente comum, com pesos fatoriais entre 0,597 e 0,797 (Tabela 5). As comunalidades variaram entre 0,356 e 0,636 .

\section{Validade convergente}

De acordo com o estudo original de Wagnild e Young ${ }^{7}$ e o guia de Wagnild, ${ }^{35}$ a resiliência deve estar correlacionada negativamente com o stress, a ansiedade e a depressão, e deve estar correlacionada positivamente com a satisfação com a vida.

Neste estudo, foram encontradas correlações significativas negativas da ER25 com a DASS-depressão ( $r$ $=-0,502 ; p<0,001)$, com a DASS-stress $(r=-0,399 ; p<$ $0,001)$ e com a DASS-ansiedade $(r=-0,341 ; p<0,001)$, e uma correlação significativa positiva com a escala de Satisfação com a Vida $(r=0,446 ; p<0,001)$, indicando validade convergente do instrumento nestes profissionais de saúde.

De igual forma, foram encontradas correlações significativas negativas da ER14 com a DASS-depressão $(r$ $=-0,502 ; p<0,001)$, com a DASS-stress $(r=-0,366 ; p<$ $0,001)$ e com a DASS-ansiedade $(r=-0,323 ; p<0,001)$, e uma correlação positiva significativa com a escala de Satisfação com a Vida $(r=0,463 ; p<0,001)$, indicando validade convergente do instrumento neste grupo de médicos.

\section{DISCUSSÃO}

No presente estudo, e com base numa amostra de médicos, os resultados encontrados na ER25 mostraram níveis de resiliência $(M=131,2)$ inferiores aos encontrados por Wagnild e Young ${ }^{7}(M=147,9)$ e por Deep e Leal ${ }^{41}(M=$

Tabela 2 - Correlações item-total corrigidas para os 25 itens da Escala de Resiliência e respetivos valores de alfa de Cronbach, caso o item em casa seja excluído ( $n=511$ médicos)

\begin{tabular}{|c|c|c|c|}
\hline & & $\begin{array}{l}\text { Correlação de item } \\
\text { total corrigida }\end{array}$ & $\begin{array}{l}\text { Alfa de Cronbach se } \\
\text { o item for excluído }\end{array}$ \\
\hline 1. & Quando faço planos, levo-os até ao fim. & 0,584 & 0,935 \\
\hline 2. & Costumo resolver o que preciso, de uma forma ou de outra. & 0,654 & 0,934 \\
\hline 3. & Sou capaz de contar comigo próprio/a, mais do que a maioria das pessoas. & 0,556 & 0,935 \\
\hline 4. & Para mim é importante manter o interesse nas coisas. & 0,575 & 0,935 \\
\hline 5. & Quando necessário, sou capaz de ficar por minha conta. & 0,634 & 0,934 \\
\hline 6. & Sinto-me orgulhoso/a por ter conseguido coisas na vida. & 0,661 & 0,934 \\
\hline 7. & Normalmente levo as coisas "a eito". & 0,526 & 0,935 \\
\hline 8. & Estou bem comigo mesmo/a. & 0,691 & 0,933 \\
\hline 9. & Sinto que sou capaz de lidar com várias coisas ao mesmo tempo. & 0,699 & 0,933 \\
\hline 10. & Sou uma pessoa determinada. & 0,729 & 0,933 \\
\hline & Raramente me questiono sobre o sentido das coisas. & 0,320 & 0,939 \\
\hline & Vivo a vida um dia de cada vez. & 0,396 & 0,938 \\
\hline 13. & Sei que consigo superar tempos difíceis porque já passei por dificuldades antes. & 0,572 & 0,935 \\
\hline 14. & Sou uma pessoa autodisciplinada. & 0,575 & 0,935 \\
\hline & Mantenho-me interessado/a nas coisas. & 0,728 & 0,933 \\
\hline & Sou capaz de me rir das coisas. & 0,643 & 0,934 \\
\hline & O facto de acreditar em mim ajuda-me a superar momentos difíceis. & 0,737 & 0,932 \\
\hline & Em situações de emergência. sou alguém em quem se pode confiar. & 0,620 & 0,934 \\
\hline & Normalmente consigo olhar para uma situação sob várias perspetivas. & 0,654 & 0,934 \\
\hline 20. & Por vezes obrigo-me a fazer coisas. quer queira ou não queira. & 0,485 & 0,936 \\
\hline 21. & A minha vida tem sentido. & 0,654 & 0,934 \\
\hline & Não costumo cismar sobre coisas em relação às quais nada posso fazer. & 0,482 & 0,937 \\
\hline & Quando me encontro numa situação difícil, costumo conseguir sair dela. & 0,728 & 0,933 \\
\hline & Tenho energia suficiente para fazer tudo o que tenho para fazer. & 0,581 & 0,935 \\
\hline & Sou capaz de me adaptar facilmente a situações imprevistas. & 0,632 & 0,934 \\
\hline
\end{tabular}


Tabela 3 - Composição dos três fatores da ER25, com indicação dos pesos fatoriais de cada item, obtidos pela ACP ( $\mathrm{n}=511$ médicos)

\begin{tabular}{|c|c|c|c|}
\hline Itens & $\begin{array}{l}\text { Pesos } \\
\text { fator } 1\end{array}$ & $\begin{array}{l}\text { Pesos } \\
\text { fator } 2\end{array}$ & $\begin{array}{l}\text { Pesos } \\
\text { fator } 3\end{array}$ \\
\hline 1. Quando faço planos, levo-os até ao fim. & 0,763 & & \\
\hline 2. Costumo resolver o que preciso, de uma forma ou de outra. & 0,751 & & \\
\hline 3. Sou capaz de contar comigo próprio/a, mais do que a maioria das pessoas. & 0,612 & & \\
\hline 6. Sinto-me orgulhoso/a por ter conseguido coisas na vida. & 0,605 & & \\
\hline 7. Normalmente levo as coisas "a eito". & 0,706 & & \\
\hline 10. Sou uma pessoa determinada. & 0,687 & & \\
\hline 14. Sou uma pessoa autodisciplinada. & 0,492 & & \\
\hline 4. Para mim é importante manter o interesse nas coisas. & 0,636 & 0,404 & \\
\hline 5. Quando necessário, sou capaz de ficar por minha conta. & 0,568 & 0,517 & \\
\hline 9. Sinto que sou capaz de lidar com várias coisas ao mesmo tempo. & 0,535 & & 0,469 \\
\hline 17. O facto de acreditar em mim ajuda-me a superar momentos difíceis. & 0,486 & 0,427 & 0,427 \\
\hline 15. Mantenho-me interessado/a nas coisas. & 0,480 & 0,415 & 0,435 \\
\hline 13. Sei que consigo superar tempos difíceis porque já passei por dificuldades antes. & & 0,587 & \\
\hline 16. Sou capaz de me rir das coisas. & & 0,601 & \\
\hline 18. Em situações de emergência, sou alguém em quem se pode confiar. & & 0,696 & \\
\hline 19. Normalmente consigo olhar para uma situação sob várias perspetivas. & & 0,727 & \\
\hline 20. Por vezes obrigo-me a fazer coisas, quer queira ou não queira. & & 0,661 & \\
\hline 25. Não tenho problema com o facto de haver pessoas que não gostam de mim. & & 0,570 & 0,462 \\
\hline 23. Quando me encontro numa situação difícil, costumo conseguir sair dela. & & 0,529 & 0,401 \\
\hline 22. Não costumo cismar sobre coisas em relação às quais nada posso fazer. & & & 0,664 \\
\hline 24. Tenho energia suficiente para fazer tudo o que tenho para fazer. & & & 0,666 \\
\hline 12. Vivo a vida um dia de cada vez. & & & 0,514 \\
\hline 8. Estou bem comigo mesmo/a. & 0,494 & & 0,627 \\
\hline 21. A minha vida tem sentido. & 0,400 & & 0,466 \\
\hline
\end{tabular}

$132,4)$ em adultos portugueses, mas superiores aos encontrados em adolescentes portugueses por Felgueiras et a/ ${ }^{39}$ $(\mathrm{M}=126,7)$.

Quanto à validação psicométrica, salienta-se o $\alpha$ de 0,941 para 23 itens (com remoção dos itens 11 e 12, por apresentarem pesos fatoriais inferiores a 0,40), e de 0,923 para 14 itens (ER14), sugerindo excelente consistência interna, fiabilidade estável e homogeneidade. Esse valor está acima dos valores apresentados por Wagnild e Young ${ }^{7}(\alpha=$ $0,91)$, por Felgueiras et $a^{\beta 9}(\alpha=0,82)$, Oliveira e Machado ${ }^{40}$ $(\alpha=0,89)$ e Deep e Leal ${ }^{41}(\alpha=0,87)$.

Embora as várias adaptações da ER25 permitam confirmar a boa consistência interna deste instrumento, no que diz respeito à sua estrutura fatorial permanecem algumas controvérsias. Há estudos que apontam para cinco fatores, ${ }^{40}$ quatro, ${ }^{41}$ três, ${ }^{53}$ para dois ${ }^{7}$ e para um fator. ${ }^{34,35}$ No presente estudo, da interpretação dos três fatores que resultaram da rotação varimax, não se distingue de forma clara 'competência pessoal' de 'satisfação de si e da vida', ${ }^{7}$ nem se adequam a outras propostas teóricas realizadas pelas autoras, ${ }^{7,35}$ pelo que se tomou a decisão de forçar a análise a um só fator, tendo esta explicado $45,27 \%$ da variân- cia total. É de salientar ainda que, apesar da proposta de solução de dois fatores das autoras ${ }^{7,35}$ da ER, as mesmas utilizam em geral as pontuações globais da escala, ${ }^{7,35}$ tanto no que se refere à ER25 como à ER14.

Como era de esperar, a versão ER25 e a versão ER14 estão altamente correlacionadas $(r=0,956 ; p<0,001)$, replicando a descoberta de Wagnild e Young. ${ }^{7}$

Quanto à validade convergente, esta foi demonstrada por correlações positivas significativas entre as duas versões de ER e a escala de Satisfação com a Vida, e por correlações negativas significativas com a depressão, a ansiedade e o stress, confirmando a proposta teórica. ${ }^{7}$ Desta forma, verificou-se que as correlações entre a ER25 e a ER14 e os construtos (ansiedade, depressão, stress e satisfação com a vida) teoricamente ligados à resiliência, foram na direção esperada e apresentaram-se estatisticamente significativas $(p<0,001)$. Por fim, é de destacar que uma das correlações mais elevadas foi com a escala de Satisfação de Vida, em que o indivíduo avalia a sua perceção de satisfação com a vida independentemente dos eventos de vida vivenciada. A resultados idênticos chegaram Pesce et a/53 e Wagnild e Young. ${ }^{7}$ 
Tabela 4 - Pesos fatoriais dos itens da ER25 na ACP com 1 fator, em três situações: A - considerando os 25 itens; B - excluindo o item 11; e $\mathrm{C}$ - excluindo os itens 11 e 12 ( $\mathrm{n}=511$ médicos).

\begin{tabular}{|c|c|c|c|}
\hline Itens & $\begin{array}{c}\text { A } \\
\text { pesos fator } 1\end{array}$ & $\begin{array}{c}\text { B } \\
\text { pesos fator } 1\end{array}$ & $\begin{array}{c}C \\
\text { pesos fator } 1\end{array}$ \\
\hline 1. Quando faço planos, levo-os até ao fim. & 0,635 & 0,637 & 0,642 \\
\hline 2. Costumo resolver o que preciso, de uma forma ou de outra. & 0,707 & 0,710 & 0,714 \\
\hline 3. Sou capaz de contar comigo próprio/a, mais do que a maioria das pessoas. & 0,613 & 0,616 & 0,620 \\
\hline 4. Para mim é importante manter o interesse nas coisas. & 0,642 & 0,649 & 0,653 \\
\hline 5. Quando necessário, sou capaz de ficar por minha conta. & 0,687 & 0,691 & 0,692 \\
\hline 6. Sinto-me orgulhoso/a por ter conseguido coisas na vida. & 0,708 & 0,708 & 0,710 \\
\hline 7. Normalmente levo as coisas "a eito". & 0,578 & 0,579 & 0,582 \\
\hline 8. Estou bem comigo mesmo/a. & 0,719 & 0,716 & 0,714 \\
\hline 9. Sinto que sou capaz de lidar com várias coisas ao mesmo tempo. & 0,734 & 0,732 & 0,732 \\
\hline 10. Sou uma pessoa determinada. & 0,776 & 0,776 & 0,780 \\
\hline 11. Raramente me questiono sobre o sentido das coisas. & 0,326 & ------- & ------- \\
\hline 12. Vivo a vida um dia de cada vez. & 0,404 & 0,397 & ------ \\
\hline 13. Sei que consigo superar tempos difíceis porque já passei por dificuldades antes. & 0,607 & 0,608 & 0,600 \\
\hline 14. Sou uma pessoa autodisciplinada. & 0,613 & 0,613 & 0,613 \\
\hline 15. Mantenho-me interessado/a nas coisas. & 0,765 & 0,767 & 0,766 \\
\hline 16. Sou capaz de me rir das coisas. & 0,685 & 0,686 & 0,684 \\
\hline 17. O facto de acreditar em mim ajuda-me a superar momentos difíceis. & 0,773 & 0,772 & 0,774 \\
\hline 18. Em situações de emergência, sou alguém em quem se pode confiar. & 0,673 & 0,676 & 0,678 \\
\hline 19. Normalmente consigo olhar para uma situação sob várias perspetivas. & 0,697 & 0,698 & 0,699 \\
\hline 20. Por vezes obrigo-me a fazer coisas, quer queira ou não queira. & 0,530 & 0,531 & 0,528 \\
\hline 21. A minha vida tem sentido. & 0,693 & 0,691 & 0,693 \\
\hline 22. Não costumo cismar sobre coisas em relação às quais nada posso fazer. & 0,500 & 0,493 & 0,486 \\
\hline 23. Quando me encontro numa situação difícil, costumo conseguir sair dela. & 0,761 & 0,762 & 0,763 \\
\hline 24. Tenho energia suficiente para fazer tudo o que tenho para fazer. & 0,604 & 0,600 & 0,597 \\
\hline 25. Não tenho problema com o facto de haver pessoas que não gostam de mim. & 0,662 & 0,661 & 0,657 \\
\hline
\end{tabular}

Tabela 5 - Pesos fatoriais dos 14 itens da ER14, obtidos pela ACP a 1 fator ( $\mathrm{n}=511$ médicos).

\begin{tabular}{lc}
\hline Itens & Pesos fator \\
\hline 2. & 0,697 \\
6. Sinto-me orgulhoso/a por ter conseguido coisas na vida. & 0,722 \\
7. Normalmente levo as coisas "a eito". & 0,597 \\
8. Estou bem comigo mesmo/a. & 0,742 \\
9. Sinto que sou capaz de lidar com várias coisas ao mesmo tempo. & 0,740 \\
10. Sou uma pessoa determinada. & 0,797 \\
13. Sei que consigo superar tempos difíceis porque já passei por dificuldades antes. & 0,603 \\
14. Sou uma pessoa autodisciplinada. & 0,640 \\
15. Mantenho-me interessado/a nas coisas. & 0,786 \\
16. Sou capaz de me rir das coisas. & 0,691 \\
17. O facto de acreditar em mim ajuda-me a superar momentos difíceis. & 0,795 \\
18. Em situações de emergência, sou alguém em quem se pode confiar. & 0,666 \\
21. A minha vida tem sentido. & 0,718 \\
23. Quando me encontro numa situação difícil, costumo conseguir sair dela. & 0,754 \\
\hline
\end{tabular}

\section{CONCLUSÃO}

A Escala de Resiliência (ER25 e ER14) avalia a capacidade de o indivíduo suportar os fatores de stress, de prosperar e dar sentido a desafios vitais. Com este instrumento é possível realizar uma avaliação desta capacidade e fornecer informação relevante ao indivíduo. De igual 
forma, o estudo da resiliência permite identificar fatores de proteção que podem orientar programas de intervenção com vista ao desenvolvimento de competências para ajudar o indivíduo a lidar com a adversidade.

Com base nas propriedades psicométricas obtidas, este estudo apoia a utilidade da ER25 (versão portuguesa longa) e ER14 (versão portuguesa breve) para avaliar a resiliência em médicos no contexto português.

Este estudo permitiu verificar as boas qualidades psicométricas da escala ER25 e ER14 numa amostra portuguesa de médicos. Recomenda-se a replicação deste estudo e a aplicação desta versão a outros profissionais de saúde, especialmente aqueles que desenvolvem a sua atividade profissional em contextos de maior adversidade, com vista a avaliar as suas características psicométricas, comparando-as com os dados aqui descritos.

\section{CONTRIBUTO DOS AUTORES}

CS: Conceção e desenho do manuscrito, aquisição, análise e interpretação dos dados, supervisão e revisão do manuscrito.

LC: Análise estatística, aquisição, análise e interpretação dos dados, revisão do manuscrito.

AT: Análise estatística, aquisição, análise e interpretação dos dados, revisão do manuscrito.

ARR: Análise e interpretação dos dados e revisão do

\section{REFERÊNCIAS}

1. Hjemdal $O$, Aune $T$, Reinfjell $T$, Stiles $T$, Friborg $O$. Resilience as a predictor of depressive symptoms: a correlational study with young adolescents. Clin Child Psychol Psychiatry. 2007;12:91-104.

2. Cordovil C, Crujo M, Vilariça $P$, Caldeira da Silva P. Resiliência em crianças e adolescentes institucionalizados. Acta Med Port. 2011;24:S413-8.

3. Rushton C, Batcheller J, Schroeder K, Donohue P. Burnout and resilience among nurses practicing in high-intensity settings. Am J Crit Care. 2015;24: 412-20.

4. American Psychological Association. The road to resilience. Washington: American Psychological Association; 2014.

5. Southwick SM, Bonanno GA, Masten AS, Panter-Brick C, Yehuda R. Resilience definitions, theory, and challenges: Interdisciplinary perspectives. Eur J Psychotraumatol. 2014;5:1-14.

6. Melillo A, Ojeda E. Resiliência: descobrindo as próprias fortalezas. Porto Alegre: Artmed; 2005.

7. Wagnild G, Young $\mathrm{H}$. Development and psychometric evaluation of the resilience scale. J Nurs Meas. 1993;1:165-78.

8. Poletto $\mathrm{M}$, Koller $\mathrm{SH}$. Contextos ecológicos: promotores de resiliência, fatores de risco e de proteção. Estud Psicol. 2008;25:405-16.

9. Norris FH, Friedman MJ, Watson PJ, Byrne CM, Diaz E, Kaniasty K. 60,000 disaster victims speak: part I. An empirical review of the empirical literature, 1981-2001. Psychiatry. 2002;65:207-39.

10. Angst R. Psicologia e resiliência: uma revisão da literatura. Psicol Argum. 2009;27:253-60.

11. Serrão C, Duarte I, Castro L, Teixeira A. Burnout and depression in Portuguese healthcare workers during the COVID-19 pandemic-the mediating role of psychological resilience. Int $\mathrm{J}$ Environ Res Public Health 2021;18:636-50.

12. Pietrzak RH, Southwick SM. Psychological resilience in OEF-OIF veterans: application of a novel classification approach and examination of demographic and psychosocial correlates. J Affect Disord. 2011;133:560-8.

13. McCain RS, McKinley N, Dempster M, Campbell WJ, Kirk SJ. A study of the relationship between resilience, burnout and coping strategies in doctors. Postgrad Med J. 2018;94:43-7.

14. McKinley N, Karayiannis PN, Convie L, Clarke M, Kirk SJ, Campbell manuscrito.

ID: Conceção e desenho do manuscrito, obtenção de financiamento, supervisão e revisão do manuscrito.

\section{PROTEÇÃO DE PESSOAS E ANIMAIS}

As autoras declaram que os procedimentos seguidos estavam de acordo com os regulamentos estabelecidos pelos responsáveis da Comissão de Investigação Clínica e Ética e de acordo com a Declaração de Helsínquia da Associação Médica Mundial atualizada em 2013.

\section{CONFIDENCIALIDADE DOS DADOS}

As autoras declaram ter seguido os protocolos da Faculdade de Medicina da Universidade do Porto acerca da publicação de dados.

\section{CONFLITOS DE INTERESSE}

As autoras declaram não ter conflitos de interesse relacionados com o presente trabalho.

\section{FONTES DE FINANCIAMENTO}

Este trabalho foi financiado por fundos nacionais através da FCT - Fundação para a Ciência e a Tecnologia, I.P., no âmbito do projeto UIDB/05198/2020 (Centro de Investigação e Inovação em Educação, inED).

WJ. Resilience in medical doctors: a systematic review. Postgrad Med J. 2019;95:140-7.

15. McKinley N, McCain RS, Convie L, Clarke M, Dempster M, Campbell WJ, et al. Resilience, burnout and coping mechanisms in UK doctors: a cross-sectional study. BMJ Open. 2020;10:1-8.

16. Creedy DK, Sidebotham M, Gamble J, Pallant J, Fenwick J. Prevalence of burnout, depression, anxiety and stress in Australian midwives: a cross-sectional survey. BMC Pregnancy Childbirth. 2017;17:1-8.

17. Yahaya S, Wahab S, Yusoff M, Yasin M, Rahman M. Prevalence and associated factors of stress, anxiety and depression among emergency medical officers in Malaysian hospitals. World J Emerg Med. 2018;9:17886.

18. Wilkinson E. UK NHS staff: stressed, exhausted, burnt out. Lancet. 2015;385:841-2.

19. Karatoreos IN, Mcewen BS. Annual research review: the neurobiology and physiology of resilience and adaptation across the life course. $J$ Child Psychol Psychiatry. 2013;54:337-47.

20. Russo S, Murrough J, Han M, Charney S, Nestler E. Neurobiology of resilience. Nat Neurosci. 2012;15:1475-84.

21. Southwick $S$, Charney D. The science of resilience: Implications for the prevention and treatment of depression. Science. 2012;338:79-82.

22. Garbarino S, Lanteri P, Durando P, Magnavita N, Sannita WG. Comorbidity, mortality, quality of life and the healthcare/welfare/social costs of disordered sleep: a rapid review. Int J Environ Res Public Health. 2016;13:1-15.

23. Lambert V, Lambert C, Itano J, Inouye J, Kim S, Kuniviktikul W, et al. Cross-cultural comparison of workplace stressors, ways of coping and demographic characteristics as predictors of physical and mental health among hospital nurses in Japan, Thailand, South Korea and the USA (Hawaii). Int J Nurs Stud. 2004;41:671-84.

24. Lim J, Hepworth J, Bogossian F. A qualitative analysis of stress, uplifts and coping in the personal and professional lives of Singaporean nurses. J Adv Nurs. 2011;67:1022-33.

25. Jackson D, Firtko A, Edenborough M. Personal resilience as a strategy for surviving and thriving in the face of workplace adversity: a literature review. J Adv Nurs. 2007;60:1-9.

26. Stamm BH. The Concise ProQOL Manual. $2^{\text {nd }}$ ed. Pocatello: ProQOL. 
org; 2010.

27. Barnett J, Baker E, Elman N, Schoener G. In pursuit of wellness: the self-care imperative. Prof Psychol Res Pract. 2007;38:603-12.

28. Schilling E, Aseltine R, Gore S. The impact of cumulative childhood adversity on young adult mental health: measures, models, and interpretations. Soc Sci Med. 2008;66:1140-51.

29. Keeton K, Fenner D, Johnson T, Hayward R. Predictors of physician career satisfaction, work-life balance, and burnout. Obstet Gynecol. 2007;109:949-55.

30. Arrogante O, Aparicio-Zaldivar E. Burnout and health among critical care professionals: the mediational role of resilience. Intensive Crit Care Nurs. 2017;42:110-5.

31. Colville G, Smith J, Brierley J, Citron K, Nguru N, Shaunak P, et al. Coping with staff burnout and work-related posttraumatic stress in intensive care. Pediatr Crit Care Med. 2017; 18:e267-73.

32. Almeida $\mathrm{M}$, Dias $\mathrm{S}$, Zavier $\mathrm{M}$, Torgal J. Validação exploratória e confirmatória da escala de resiliência Connor-Davidson (CD-RISC-10) numa amostra de inscritos em centros de emprego. Acta Med Port. 2020;33:124-32.

33. Wagnild G, Young $H$. Resilience among older women. Image J Nurs Scholarsh. 1990;22:252-5.

34. Pinheiro M, Matos A. Exploring the construct validity of the two versions of the resilience scale in a Portuguese adolescent sample. EJSBS. 2013;2:178-89.

35. Wagnild G. The resilience Scale user's guide for the US English version of the resilience scale and the 14-item resilience scale. Worden: Resilience Center; 2009.

36. Wagnild G. A review of the resilience scale. J Nurs Meas. 2009;17:10513.

37. Black C, Ford-Gilboe M. Adolescent mothers: resilience, family health work and health-promoting factors. J Adv Nurs. 2004;48:351-60.

38. Hunter AJ, Chandler G. Adolescent resilience. Image J Nurs Scholarsh. 1999;31:243-47.

39. Felgueiras M, Festas $\mathrm{C}$, Vieira M. Adaptação e validação da resilience scale de Wagnild e Young para a cultura portuguesa. Cad Saúde. 2010;3:73-80.

40. Oliveira M, Machado T. Tradução e validação da escala de resiliência para estudantes do ensino superior. Anal Psicol. 2011:4:579-91.

41. Deep C, Leal I. Adaptação da "The Resilience Scale" para a população adulta portuguesa. Psicol USP. 2012;23:417 - 33

42. Streiner D, Norman R, Cairney J. Health measurement scales: a practical guide to their development and use. $5^{\text {th }}$ ed. Oxford: Oxford University Press; 2014.

43. Marôco J. Análise estatística com o SPSS statistics. $6^{\text {th }}$ ed. Pêro Pinheiro: ReportNumber. Lisboa; 2014.

44. Mokkink L, Terwee C, Patrick D, Alonso J, Stratford P, Knol D, et al. The COSMIN study reached international consensus on taxonomy, terminology, and definitions of measurement properties for healthrelated patient-reported outcomes. J Clin Epidemiol. 2010;63:737-45.

45. Nunnally JC. Psychometric theory. McGraw-Hill: New York; 1978.

46. Clarck L, Watson D. Constructing validity: basic issues in objective scale development. Psychol Assess. 1995;7:309-19.

47. Terwee C, Bot S, De Boer M, Van der Windt D, Knol D, Dekker J, et al. Quality criteria were proposed for measurement properties of health status questionnaires. J Clin Epidemiol. 2007;60:34-42,

48. Lovibond $\mathrm{P}$, Lovibond $\mathrm{S}$. The structure of negative emotional states: comparison of the depression anxiety stress scales (DASS) with the Beck depression and anxiety inventories. Behav Res Ther. 1995;33:33543.

49. Pais-Ribeiro JL, Honrado A, Leal I. Contribuição para o estudo da adaptação portuguesa das escalas de ansiedade, depressão e stress (EADS) de 21 itens de Lovibond e Lovibond. Psic Saúde Doenças. 2004;5:229-39.

50. Diener ED, Emmons RA, Larsen RJ, Griffin S. The satisfaction with life scale. J Pers Assess. 1985;49:71-5.

51. Simões A. Ulterior validação de uma escala de satisfação com a vida (SWLS). Rev Port Pedagogia. 1992;26:503-15.

52. Neto F, Barros J, Barros A. Satisfação com a vida. In: Almeida L, Santiago R, Silva P, Caetano O, Marques J, editors. A ação educativa: análise psicossocial. Leiria: ESEL/APPORT; 1990. p.105-17.

53. Pesce RP, Assis S, Avanci JQ, Santos NC, Malaquias JV, Carvalhaes R. Adaptação transcultural, confiabilidade e validade da escala de resiliência. Cad Saúde Pública. 2005;21:436-48. 